\title{
Future of radiation therapy for malignant melanoma in an era of newer, more effective biological agents
}

This article was published in the following Dove Press journal:

OncoTargets and Therapy

8 August 201I

Number of times this article has been viewed

\author{
Mohammad K Khan' \\ Niloufer Khan ${ }^{2}$ \\ Alex Almasan ${ }^{1,2}$ \\ Roger Macklis' \\ 'Taussig Cancer Institute, Lerner \\ College of Medicine, Cleveland Clinic, \\ Cleveland, OH, USA; ${ }^{2}$ Case Western \\ Reserve University School \\ of Medicine, Cleveland, OH, USA
}

\begin{abstract}
The incidence of melanoma is rising. The primary initial treatment for melanoma continues to be wide local excision of the primary tumor and affected lymph nodes. Exceptions to wide local excision include cases where surgical excision may be cosmetically disfiguring or associated with increased morbidity and mortality. The role of definitive or adjuvant radiotherapy has largely been relegated to palliative measures because melanoma has been viewed as a prototypical radiotherapy-resistant cancer. However, the emerging clinical and radiobiological data summarized here suggests that many types of effective radiation therapy, such as radiosurgery for melanoma brain metastases, plaque brachytherapy for uveal melanoma, intensity modulated radiotherapy for melanoma of the head and neck, and adjuvant radiotherapy for selected highrisk, node-positive patients can improve outcomes. Similarly, although certain chemotherapeutic agents and biologics have shown limited responses, long-term control for unresectable tumors or disseminated metastatic disease has been rather disappointing. Recently, several powerful new biologics and treatment combinations have yielded new hope for this patient group. The recent identification of several clinically linked melanoma gene mutations involved in mitogenactivated protein kinase (MAPK) pathway such as BRAF, NRAS, and cKIT has breathed new life into the drive to develop more effective therapies. Some of these new therapeutic approaches relate to DNA damage repair inhibitors, cellular immune system activation, and pharmacological cell cycle checkpoint manipulation. Others relate to the investigation of more effective targeting and dosing schedules for underutilized therapeutics, such as radiotherapy. This paper summarizes some of these new findings and attempts to give some context to the renaissance in melanoma therapeutics and the potential role for multimodality regimens, which include certain types of radiotherapy as aids to locoregional control in sensitive tissues.
\end{abstract}

Keywords: hypofractionation radiotherapy, brachytherapy, radiosurgery, melanoma cell cycle, targeted biologic agents, systemic agents

\section{Introduction}

Several recent reviews have discussed changes in the systemic management of melanoma, including the potential use of newer, more effective biologic agents. ${ }^{1-3}$ Because certain types of radiotherapy have also been enjoying a rebirth of enthusiasm, we have chosen to focus this review on some of the radiotherapeutic implications of multimodality treatment for a clinically heterogeneous disease. We call attention to the fact that older therapeutic modalities, such as radiation, previously consigned to the melanoma scrap heap as ineffective, may still have a significant role in the future. Furthermore, as our understanding of the biological mechanisms of melanoma tumorigenesis increases and we find ways to exploit these mechanisms biologically, it is likely that the role of radiation therapy will continue expanding beyond what is
Correspondence: Mohammad K Khan Taussig Cancer Institute, Cleveland Clinic Foundation, Cleveland, $\mathrm{OH} 44106$, USA

$\mathrm{Tel}+\mathrm{I} 2164454379$

$\mathrm{Fax}+\mathrm{I} 2164451068$

Email drkhurram2000@gmail.com 
currently being done. In addition, differences in the molecular and biological makeup of cutaneous melanomas compared with that of retinal or mucosal melanoma may also help in further tailoring radiotherapy fractionation and technical approaches in the future.

In their classic 1946 text, McKee and associates ${ }^{4}$ opine that "in spite of occasionally good results, it is our opinion that irradiation alone by any technique should not be relied on for the cure of these lesions". This reputation as one of the least radioresponsive and least radiocurable tumors has dogged melanoma since the early days of orthovoltage x-ray treatments for skin conditions. In the modern era, many investigators are beginning to question the accuracy of this reputation. ${ }^{5-9}$ In fact, in vitro data on melanoma cell lines are generally acknowledged to show high levels of DNA damage repair at conventional fractionation doses and increased cell death with larger doses per fraction. A popular hypofractionated regimen involving a dose of 7 Gy per fraction delivered on days 1,7 , and 21 for postoperative patients demonstrated complete remission rates of $40 \%$, with greater than $90 \%$ local control rates at four years. ${ }^{5}$ Some of the radioresistance based on recently reported in vivo biological data from mice suggests that a radiation-induced decrease in substance P may partly explain the radioresistant nature of some melanoma cell lines, ${ }^{10}$ and that altered fractionation regimens may be needed to overcome this based on clinical studies. ${ }^{5}$

Although the trends to utilize radiotherapy have been slow to catch on, recent evaluations suggest that the reputation of malignant melanoma as a "radioinsensitive" disease may not be entirely justified. As such, the role of radiotherapy in the future will likely continue to grow in light of novel emerging biologic agents which have yet to be investigated in the concurrent combined multimodality setting. This review summarizes some of the clinical and radiobiological evidence for the role of radiotherapy and will focus on four relatively new areas of clinical growth, ie, intracranial radiosurgery, retinal plaque brachytherapy, intensity modulated radiotherapy for mucosal melanoma of the head and neck region, and lymphatic field radiotherapy for node-positive, surgically debulked cases. For some of these clinical situations, it appears that moderate dose radiotherapy may be considered a viable option to surgery (intracranial metastases, uveal melanoma, mucosal melanoma of the head and neck) and as an adjunct in others. Here we also discuss some of the biological understanding behind malignant transformation of melanoma and then focus on some of the emerging targeted systemic options which provide increased optimism for radiotherapists in the future.

\section{Epidemiology}

Melanoma has the fastest growing incidence of any cancer among men, and the second fastest growing incidence among women. ${ }^{11}$ In 2010, melanoma will be responsible for 68,130 new cases and 8700 deaths, and is the fifth and sixth leading cause of cancer in men and women, respectively. ${ }^{12}$ The lifetime risk for the development of melanoma is one in 39 for men and one in 58 for women, with Caucasian men being at the highest risk. ${ }^{12}$ The median age at diagnosis is 59 years, peaking during the fourth and fifth decades. At diagnosis, $82 \%-85 \%$ will have localized disease, $10 \%-13 \%$ will have regional disease, and $2 \%-5 \%$ will have nonregional metastatic disease. ${ }^{13}$ The five-year survival rates are $98 \%$ if localized, $62 \%$ if regional, and $15 \%$ if metastatic. ${ }^{11}$ The fiveyear overall survival rates have increased over the decades, from $82 \%$ in $1975-77$, to $87 \%$ in $1984-86$, and to $92 \%$ in $2004 .^{12}$ The increase in overall survival is partly attributed to earlier detection as a result of increased education and public awareness. ${ }^{14}$

\section{Surgical excision}

The primary treatment of skin melanoma continues to be wide local excision. Sentinel node biopsy with lymph node dissection is reserved for advanced infiltrative lesions at high risk for spread to regional lymph nodes. Patients considered most appropriate for sentinel node biopsy include stage IB, stage II with a $\leq 1 \mathrm{~mm}$ thick lesion and ulceration or mitotic rate $\geq 1 / \mathrm{mm}^{2}$, stage II with a $>1 \mathrm{~mm}$ thick lesion, and resectable stage III tumors with in transit metastases. ${ }^{15}$ The extent of wide local excision has been evaluated in several large-scale randomized clinical trials. The controversy has revolved around the margins of excision. A trial from France determined that for melanomas with Breslow depth $<2.1 \mathrm{~mm}$, there was no difference in 10-year overall survival between a $2 \mathrm{~cm}$ and a $5 \mathrm{~cm}$ excision margin. ${ }^{16}$ The findings using narrower margins for surgical resection have been confirmed in other randomized trials ${ }^{17-19}$ as well as in a more recent study from the UK. ${ }^{20}$ These randomized studies have served as the basis for the current National Cancer Center Network guidelines ${ }^{15}$ (Table 1).

\section{Historical management: limited role for conventional radiotherapy}

Historically, melanoma was considered highly radioresistant. Simpson in 1913 was one of the first to treat a black nevus with radiation successfully and with little damage of surrounding skin. ${ }^{6}$ His approach was considered unwise due to concerns about toxicity. ${ }^{21}$ Controversy over the role of 
Table I Guidelines for surgical excision of melanoma, from the National Cancer Center Network Practice Guidelines in Oncology ${ }^{15}$

\begin{tabular}{ll}
\hline Tumor thickness & Recommended margins \\
\hline In situ & $0.5 \mathrm{~cm}$ \\
$<1.0 \mathrm{~mm}$ & $1.0 \mathrm{~cm}$ \\
$1.01-2 \mathrm{~mm}$ & $1-2 \mathrm{~cm}$ \\
$2.01-4 \mathrm{~mm}$ & $2.0 \mathrm{~cm}$ \\
$>4 \mathrm{~mm}$ & $2.0 \mathrm{~cm}$ \\
\hline
\end{tabular}

radiotherapy continued until two landmark studies provided convincing evidence to support Simpson. Ellis in 1939 treated 38 patients using $55-60$ Gy over 7-10 days and demonstrated a good response in 12 patients. ${ }^{6,22}$ The second study in 1963 reported on the 25-year experience, and demonstrated that the five-year overall survival was $68 \%$ in 95 patients, with results that equaled surgery. ${ }^{6,23}$ Despite the promise of these earlier studies, advances in infection control and anesthesia led to improved surgical morbidity and mortality, and surgery became the preferred therapy. Radiation use waned until the 1970s when reports of melanoma radiobiology suggested that melanoma cells were heterogeneous, not universally radioresistant. ${ }^{6,9,24}$

The first prospective randomized trial was published in 1991 by the Radiation Therapy Oncology Group (RTOG $83-05) .{ }^{25,26}$ The trial tested the earlier radiobiological findings that a higher radiation dose per treatment is needed to control melanoma by comparing a hypofractionated group receiving 8 Gy per fraction in four treatments delivered over 21 days with a standard group receiving 2.5 Gy per fraction in 20 treatments delivered five days a week over 26-28 days. The trial demonstrated an overall response rate of $57 \%-60 \%$, but failed to show a difference between the different fractionation regimens. Furthermore, the overall response rate of around $60 \%$ was much lower than the previously reported response rates of $97 \%$ comparing 9 Gy in three fractions with $5 \mathrm{~Gy}$ in eight fractions delivered twice a week..$^{25}$ Thus, RTOG 83-05 was closed prematurely.

In contrast with RTOG 83-05, several retrospective studies have reported that a large fraction size was linked to improved response rates, confirming earlier radiobiological findings. ${ }^{27}$ One study of 35 patients (67 cutaneous or lymph node metastases) showed a complete response of $9 \%$ if $\leq 5$ Gy per treatment was used and $50 \%$ if radiation doses $\geq 5$ Gy per treatment were used..$^{28}$ Another study from 1983-1988 demonstrated 2-year locoregional control of $95 \%$ in head and neck melanoma patients presenting with clinically negative lymph nodes treated with larger doses of adjuvant radiation (24-30 Gy in 4-5 fractions at 5-6 Gy per fraction) to the tumor bed and regional lymph nodes. The authors concluded that locoregional control rates were better than surgery alone for comparable patients and that treatment morbidity with the addition of hypofractionated radiation was minimal. ${ }^{29}$

\section{Recent growth in role of radiotherapy}

Since the 1970s, radiotherapy has been used more frequently to treat melanoma, particularly in areas where surgical excision has had limited success for cosmetic reasons or morbidity of the surgical procedure itself. Areas of recent growth include radiosurgery for brain metastases, plaque brachytherapy for uveal melanoma, and intensity modulated radiotherapy for head and neck melanoma. Furthermore, there is likely to be an increase in the use of adjuvant radiotherapy for selected high-risk patients with recurrent nodepositive disease based on newer clinical data which suggests improved progression-free survival with the use of postoperative radiotherapy for selected high-risk patients. ${ }^{30,31}$

We have already witnessed a change in the referral patterns at our institution. This growth is a direct result of greater clinical and radiobiological understanding of the response of melanoma to radiotherapy, and delivering hypofractionated radiotherapeutic regimens in a much more conformal way. As we continue to learn more about the differences in the natural history and biology of retinal versus cutaneous and mucosal melanoma, radiotherapy may continue to be further refined in the future. For example, the natural history of uveal melanoma dictates radiotherapy focused only to the primary site, whereas radiotherapy for mucosal melanoma of the head and neck region may dictate radiotherapy to the primary site as well as the regional lymphatics. Lastly, the use of local radiotherapy only to the primary site versus treating the local site plus the lymphatic regions for cutaneous melanoma is a function of stratifying patients into low risk versus high risk for regional recurrence based on particular clinical and biological indicators.

\section{Radiosurgery and whole brain radiation}

The brain is a common site of melanoma metastasis, with brain metastases contributing to $20 \%-54 \%$ of all deaths. ${ }^{32}$ Patients at increased risk for brain metastases include males, patients with head and neck mucosal melanomas, and lesions with metastases to $\geq 3$ regional lymph nodes. ${ }^{33}$ These late-stage patients with brain metastasis generally have a poor prognosis, and treatment options are limited. Chemotherapy is generally ineffective. However, radiotherapy can 
get beyond the meninges and provide targeted high-dose treatment, making it a valuable weapon in the arsenal against melanoma brain metastases.

Radiotherapy options for brain metastases include stereotactic radiosurgery alone, whole brain radiotherapy with or without stereotactic radiosurgery, and postoperative whole brain radiotherapy. Several retrospective studies have shown the efficacy of both linear accelerator-based stereotactic radiosurgery (LINAC-SRS) as well as gamma knife-based radiosurgery. These studies are summarized in Tables 2 and 3 and have demonstrated 1-year local control rates of $49 \%-90 \%$ with both LINAC-SRS and gamma knife-based radiosurgery. ${ }^{34-37}$ Stereotactic radiosurgery also improves quality of life while reducing symptoms such as headaches, seizures, and visual disturbances. ${ }^{38}$ Lastly, stereotactic radiosurgery may also improve overall survival by 7.3 months when combined with whole brain radiotherapy. ${ }^{39}$

\section{Plaque brachytherapy for uveal melanoma}

Uveal melanomas arise in the choroid, ciliary body, or iris. Their molecular pathogenesis is distinct from that of the cutaneous melanomas. Instead of a BRAF, NRAS, or cKIT mutation, $83 \%$ of uveal melanomas have a mutation at the GNA11 or GNAQ loci. ${ }^{40,41}$ These genes encode heterotrimeric $\mathrm{G}$ proteins which, through downstream effects, upregulate the mitogen-activated protein kinase pathway in melanocytes when activated. G proteins generated from the mutant genes lose their GTPase activity and are constitutionally

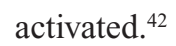

Historically, uveal melanomas were treated with enucleation. The Collaborative Ocular Melanoma Study randomized 1317 patients with medium-sized choroidal melanomas (2.5-10.0 $\mathrm{mm}$ in apical height and $5-16 \mathrm{~mm}$ in largest basal diameter) to enucleation or iodine- 125 brachytherapy and found no difference in 5-year survival rates $(81 \%$ for enucleation and $82 \%$ for brachytherapy, $P=0.48) .{ }^{43}$ Plaque brachytherapy (Figure 1) has evolved into a promising alternative to enucleation by providing equivalent overall survival with a better chance of long-term visual preservation and improved cosmetic outcomes.

The next step in uveal melanoma treatment may be the use of novel biologic agents. Just as the BRAF inhibitor, PLX4032, successfully treated patients with cutaneous melanomas, a MEK inhibitor may produce similar tumor shrinkage and improve the survival of uveal melanoma patients. ${ }^{40}$ The role of a MEK inhibitor when combined concurrently with radiation therapy is yet to be investigated in a randomized clinical trial.

\section{Mucosal head and neck melanoma}

Primary mucosal melanomas of the head and neck region have less than a $30 \% 5$-year survival rate. ${ }^{44} \mathrm{~A}$ retrospective review of 48 patients treated with surgery alone, surgery and adjuvant radiotherapy, or surgery and biochemotherapy (with or without adjuvant radiotherapy) showed that radiation therapy decreased local failure rates, but without impacting overall survival. The lack of benefit in overall survival was due to the high rate of distant metastases. ${ }^{45}$ Another retrospective review of 69 patients, with 23\% reporting lymph node involvement, reviewed the results of 30 patients who had surgery alone and 39 patients who had postoperative radiotherapy ( 70 Gy in 29 patients and 50 Gy in 10 patients). Local control was improved in patients who received adjuvant radiation treatment, but survival rates were worse. The patients who received radiotherapy developed significantly more systemic metastases, but on multivariate analysis this was ascribed to a more advanced tumor and nodal classification in the radiotherapy group. ${ }^{44}$

Recent case reports have addressed the differences in molecular mutations between melanomas occurring in chronic sun exposed regions (infrequent BRAF and NRAS and increased cKIT mutation) compared with those in regions not exposed to sun, ${ }^{46}$ and have suggested that treatment with imatinib, a c-KIT inhibitor, may provide additional optimism. ${ }^{47}$

Table 2 Role of linear accelerator stereotactic radiosurgery in the treatment of melanoma brain metastases

\begin{tabular}{|c|c|c|c|c|}
\hline Study & Patients (n) & One-year LC & One-year OS & Comments \\
\hline \multirow[t]{2}{*}{ Mori et a $\left.\right|^{34}$} & 60 & $90 \%$ & 7 months & $\begin{array}{l}\text { Improved survival on multivariate analysis included lack of } \\
\text { active systemic disease and at least one metastasis. }\end{array}$ \\
\hline & $5 I($ WBRT + SRS) & & & \\
\hline \multirow[t]{4}{*}{ Selek et $\mathrm{al}^{35}$} & 103 & $49 \%$ & 6.7 months & $75 \%$ LC for tumors $<2 \mathrm{~cm}$ with initial SRS alone \\
\hline & $6 \mathrm{I}(\mathrm{SRS})$ & $60 \%$ & 7.5 months & \\
\hline & $12(\mathrm{SRS}+\mathrm{WBRT})$ & $0 \%$ & 3.7 months & \\
\hline & 30 (SRS after WBRT) & $37 \%$ & 5.4 months & \\
\hline
\end{tabular}

Abbreviations: LC, local control; OS, overall survival; SRS, stereotactic radiosurgery; WBRT, whole brain radiotherapy. 
Table 3 Role of gamma knife-based radiosurgery in the treatment of melanoma brain metastases

\begin{tabular}{lllll}
\hline Study & Patients $(\mathbf{n})$ & One-year LC & One-year OS & Comments \\
\hline Yu et al $^{36}$ & 122 & $90 \%$ & 7 months & $\begin{array}{l}\text { Improved survival on multivariate analysis }=\text { total intracranial } \\
\text { tumor volume }<3 \mathrm{~cm}^{3}, \text { inactive systemic disease }\end{array}$ \\
Radbill et $\mathrm{al}^{37}$ & $5 \mathrm{I}$ & $81 \%$ & 26 weeks & \\
& GKRS alone $=32$ & & & \\
\hline
\end{tabular}

Abbreviations: GKRS, gamma knife-based radiosurgery; OS, overall survival.

Using molecular biology to develop a tailored multidisciplinary combined approach is an area of ongoing research.

\section{Control of lymph node-positive disease}

Although earlier studies ${ }^{48}$ suggested adjuvant radiation therapy for melanoma was ineffective, newer studies suggest better local and regional control in selected high-risk patients. Patients with desmoplastic histology, positive margins, recurrent disease, and/or a $>4.0 \mathrm{~mm}$ Breslow lesion with ulceration or satellitosis are at high risk for local recurrence and may benefit from adjuvant radiotherapy to the local site. ${ }^{49}$ Patients with at least four lymph nodes, extracapsular extension, lymph node size $\geq 3 \mathrm{~cm}$, cervical lymph node involvement, sentinel lymph node involvement but without complete lymph node dissection, and recurrent disease are at high risk for nodal relapse and may benefit with radiotherapy to the nodal basins. ${ }^{49-51}$ Further progress in this arena would be welcomed, because postsurgical recurrence in the nodal basins is associated with lower survival rates. ${ }^{30}$

A Phase II study of 48 Gy in 20 fractions to the nodal basins after surgery showed impressive regional control compared with historical cohorts. ${ }^{30}$ The study enrolled

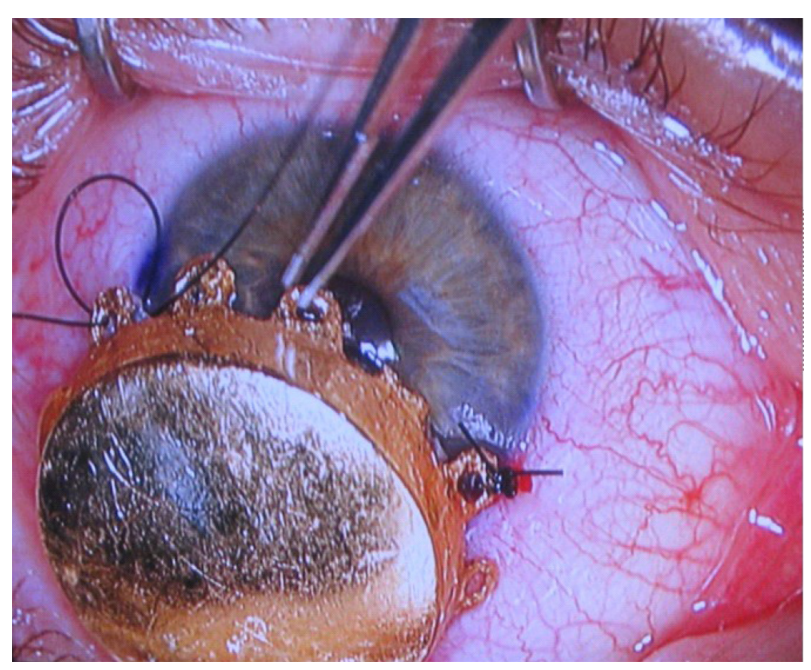

Figure I lodine ${ }^{125}$ plaque brachytherapy for a patient with an iris melanoma.
234 patients with disease in three nodal basins (head and neck, axilla/supraclavicular, and ilioinguinal). The authors demonstrated a low infield recurrence rate (7\%), a low adjacent relapse rate (14\%), and an impressive 5-year regional control rate of $91 \%$ with use of adjuvant radiotherapy. However, the 5-year overall survival rates (36\%) and progression-free survival rates (27\%) continued to be dismal due to uncontrolled systemic metastases. ${ }^{30}$ The side effects after radiotherapy were minimal and radiotherapy was well tolerated. The recommended postoperative radiation fields ${ }^{30}$ are shown in Figure 2.

This Phase II study was followed by a multicenter Phase III trial. ${ }^{31}$ The trial included post lymphadenectomy patients with isolated regional recurrence who were deemed to be high-risk $(>25 \%)$ for further regional recurrence (one or more parotid lymph nodes, at least two cervical or axillary lymph nodes, at least three groin nodes, any extranodal spread of melanoma, or maximum metastatic node diameter $\geq 3 \mathrm{~cm}$ in neck or axilla, or $\geq 4 \mathrm{~cm}$ node in the groin). Two hundred and fifty patients were randomly assigned to observation versus regional radiation therapy using 48 Gy in 20 fractions delivered at 2.4 Gy per fraction. The study showed that postoperative radiation resulted in improved disease-free survival (hazard ratio [HR] 1.77 with $P=0.041$ ). ${ }^{31}$ Others have also recommended the use of adjuvant radiotherapy after therapeutic lymphadenectomy for patients with a high risk for recurrence in the nodal basin..$^{52,53}$

\section{Malignant transformation}

Several aberrant genes are implicated in the pathogenesis of malignant melanoma: ${ }^{44,55}$ BRAF (enhances cell division); N-RAS (promotes cell proliferation); MITF overexpression (promotes survival and inappropriate cell cycle progression); c-KIT (involved in invasion and metastases); SLUG (involved in metastases); and EDNRB (involved in invasiveness). Alternatively, loss of tumor suppressor CDKN2A (encoding $\mathrm{p} 16^{\text {INK4a }}$ and ARF, that are involved in cell cycle progression), E-cadherin (CDH1, involved in tumor progression), and p53 (TP53 gene, playing a role in response to DNA damage) 


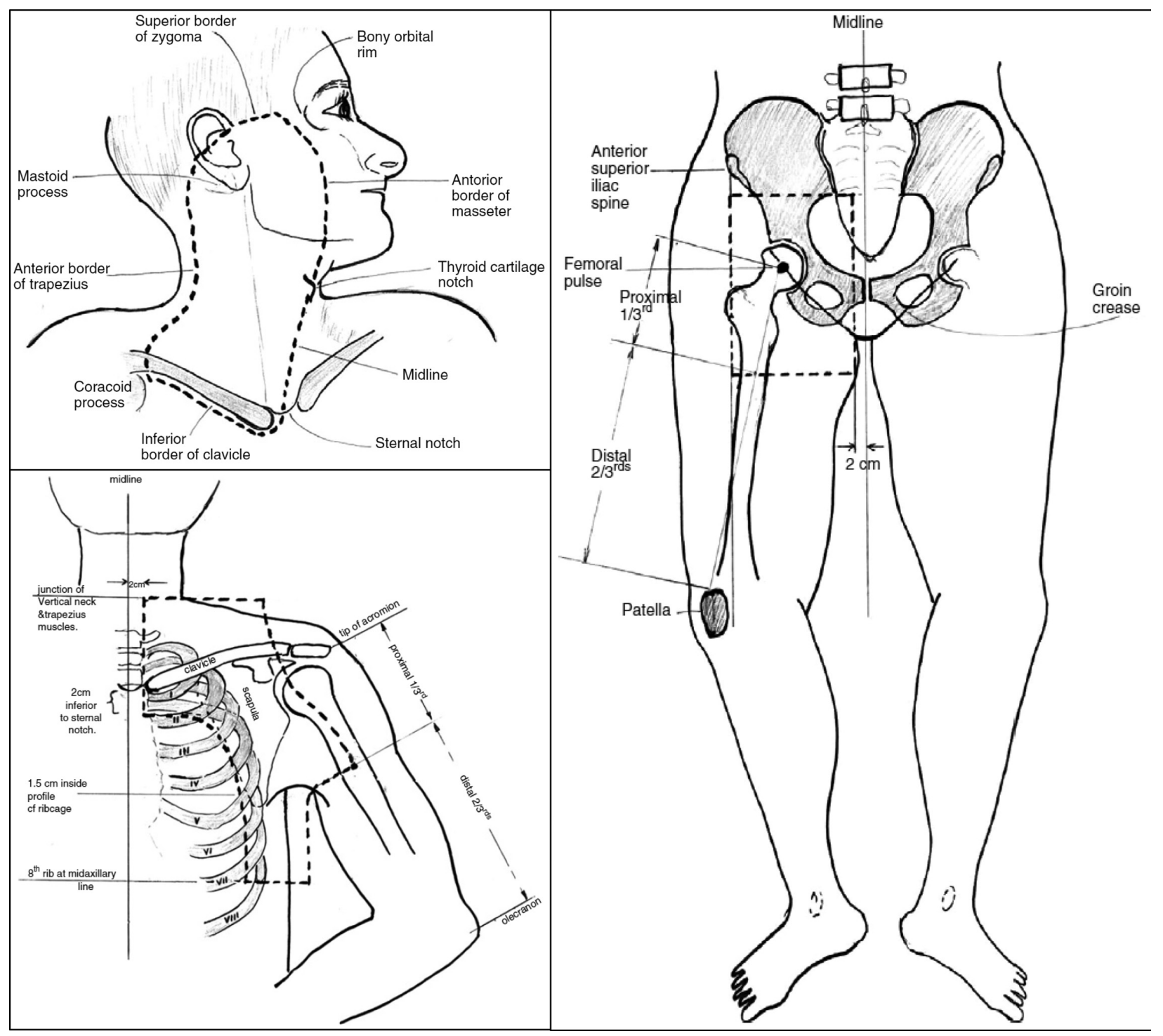

Figure 2 Post surgical radiation fields for node positive patients.

Reprinted from Burmeister BH, Mark Smithers B, Burmeister E, et al. A prospective phase II study of adjuvant postoperative radiation therapy following nodal surgery in malignant melanoma-Trans Tasman Radiation Oncology Group (TROG) Study 96.06. Radiother Oncol. 2006;81:136-I42. Copyright @ 2007, With permission from Elsevier. ${ }^{30}$

may also play a role in the radioresistance of some human melanoma cell lines..$^{56}$

The movement of cells through the cell cycle is regulated by sequential expression of cyclins and activation of their catalytic partners, cyclin-dependent kinases. For example, cyclin $\mathrm{D}$, which regulates the transition from the $\mathrm{G} 1$ to $\mathrm{S}$ phase, binds to and activates the cyclin-dependent 4 and cyclin-dependent 6 kinases. These complexes phosphorylate the retinoblastoma protein, which disassociates from E2F and allows E2F to activate transcription of critical DNA synthesis genes and cyclins, such as cyclin E, facilitating progression into the $\mathrm{S}$ phase of the cell cycle (Figure 3). ${ }^{57-59}$ Important regulators of this pathway are inhibitors of the cyclin-dependent kinases, ie, CDKN1A (p21/WAF1, activated primarily by $\mathrm{p} 53$ ) and $\mathrm{p} 16$, which complexes with cyclin-dependent kinases 4 and 6 and prevents phosphorylation of retinoblastoma protein, as well as ARF (CDKN2A), leading to inhibition of $\mathrm{p} 53$ degradation via the ubiquitin ligase $\mathrm{hDM} 2$.

An alternative pathway implicated in cell survival and increased reproduction involves the mitogen-activated protein kinase signaling cascade (Figure 3). ERK1/2 in this pathway relays the proliferative/cell survival signals via various targets that provide cross-talk with cell cycle (cyclin D, RBL2/p130, Myc) or cell survival (Bim, Mcl-1) regulators (Figure 3). ${ }^{56,60}$ The PI3K-AKT pathway is also critical, with more than $60 \%$ of human melanomas exhibiting activated AKT, and inactivation and/or deletion of the PI3K negative regulator (PTEN) 


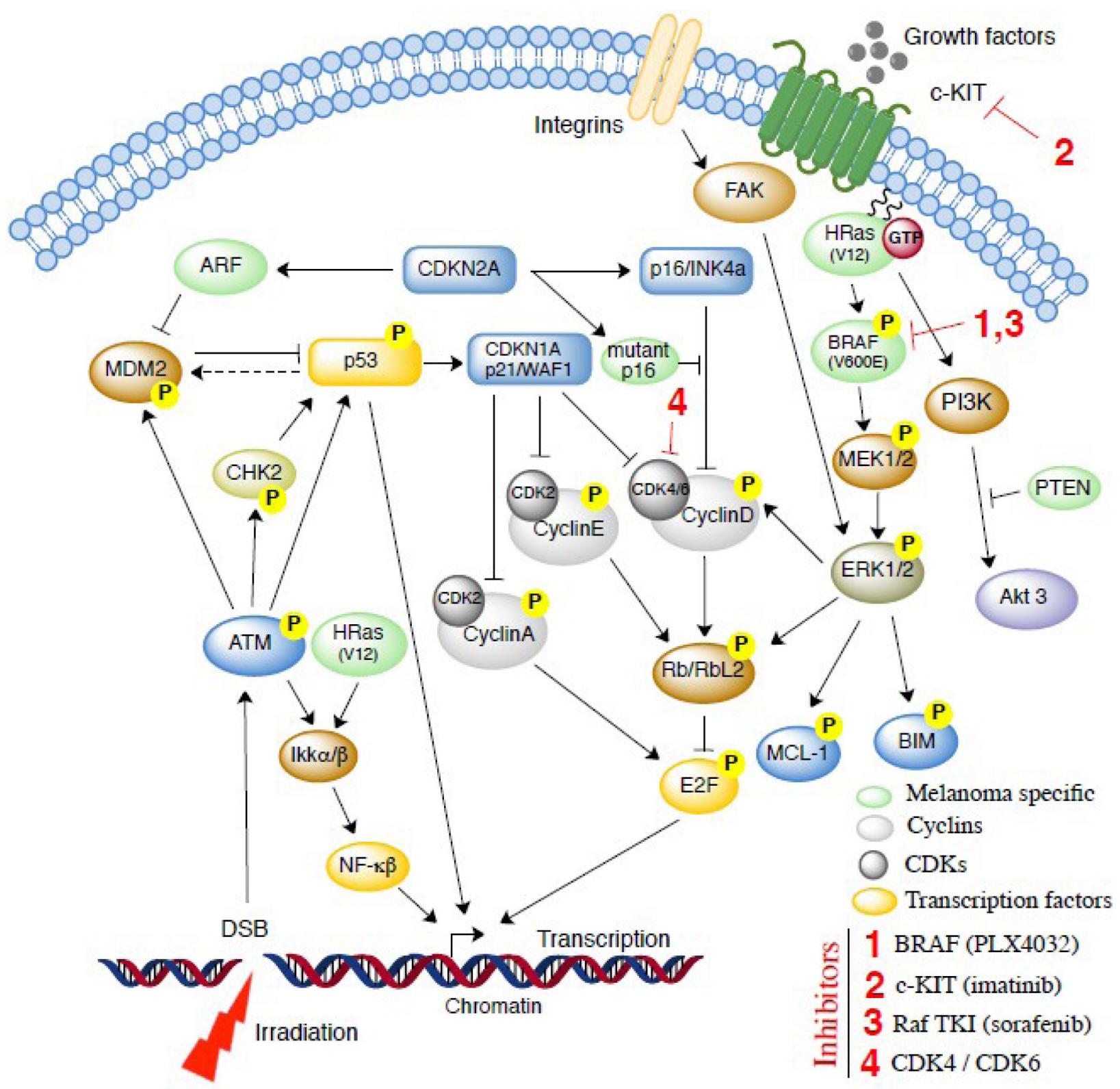

Figure 3 Cell survival signals and potential therapeutic targets for malignant melanoma.

occurring in 5\%-15\% of uncultured melanoma specimens and metastasis, $17 \%$ of short-term melanoma cultures, and $30 \%-40 \%$ of established melanoma cell lines. ${ }^{61}$

\section{Melanoma mutations: future targets and hypofractionated radiotherapy}

A better understanding of the role played by cyclin-dependent kinases in cell cycle blocks is particularly important, because these molecules are also involved in controlling and modifying the radioresponse of melanoma cells (Figure 3). By controlling cyclin-dependent kinases, one can develop a mechanism to explain the relative radioresistance of different melanoma cell lines and provide radiobiological evidence as to why either higher doses of radiation may overcome potentially lethal damage repair or how radiation may be combined with the newer biological agents.

The $\mathrm{p} 16-\mathrm{Rb}$ tumor suppressor pathway is required for the initiation and maintenance of cellular senescence. Senescence can be overcome if the pathway is not fully engaged, as may occur when p16 is inactivated. ${ }^{62}$ p16 can initiate a cyclindependent kinase 4/6-dependent autonomous senescence program that is disabled by inherited melanoma-associated mutations. ${ }^{62}$ As more knowledge is obtained regarding aberrant components of the cell cycle regulatory circuit leading to melanoma development, therapeutic trials targeting specific 
mutant proteins are getting underway. For example, one study using human melanoma cancer cell lines cultured in vitro and in mice in vivo showed that selective and structurally distinct small molecular inhibitors of cyclin-dependent kinase 4 and cyclin-dependent kinase 6 resulted in increased cellular radioresistance, especially in those cancer cell lines dependent on the cyclin-dependent kinase 4/6 pathway for proliferation. In contrast, cyclin-dependent kinase 4/6 inhibitors did not protect cell lines that proliferated independently of cyclin-dependent kinase $4 / 6$ activity. ${ }^{63}$

Sorafenib is a Raf tyrosine kinase inhibitor that inhibits the mitogen-activated protein kinase pathway (Figure 3 ) in vitro and in vivo. It has antiangiogenic effects and has been effective in treating cancers with and without BRAF mutations, making it a promising adjuvant therapy for melanoma. ${ }^{60}$ Because the majority of melanomas harbor an activating missense mutation (V600E) in the BRAF oncogene, targeted inhibition of the V600E gene product is an important therapeutic goal. ${ }^{64}$ Pharmacologic inhibition of oncogenic BRAF blocks proliferation and causes tumor regression. A Phase I trial of PLX4032, an oral inhibitor of V600E-mutated BRAF, demonstrated complete or partial tumor regression in $81 \%$ of patients and improved median progression-free survival from 2.0-6.2 months for patients with metastatic melanoma. ${ }^{65}$

Current research is exploring the use of adjuvant therapies targeted at normal cells to reduce the toxicity of cancer treatments. ${ }^{66}$ These therapies suppress apoptosis in healthy cells by inhibiting $\mathrm{p} 53$, activating NF- $\kappa \mathrm{B}$, and preventing cell cycle progression by preventing the cyclin-dependent kinases from complexing with cyclin D. ${ }^{66}$ Conversely, targeting the cell cycle control pathway components in tumor cells when combined with hypofractionated radiotherapy protocols may further improve the radioresponsiveness of melanoma. In addition to being highly active in melanoma cells, the COX-2, P13K-AKT, and NF- $\mathrm{KB}$ pathways are also involved in the radioprotective response. It has been shown recently that Ink4a/Arf-/- mice with melanocyte-specific deletion of $I k k \beta$ were protected from H-RasV12-initiated melanoma when p53 was expressed, providing genetic and mechanistic evidence that mutant $\mathrm{H}$-Ras initiation of tumorigenesis requires Ikk $\beta$-mediated NF- $\kappa B$ activity. ${ }^{67}$ Suppressing those pathways pushes higher numbers of melanoma cells into the mitotic phase, which is the most radiosensitive phase in the cell cycle, leading to decreased overall melanoma cell survival. ${ }^{68}$ One study compared the radiosensitivity of cells expressing wild-type p16 with those having the mutant p16 found in melanomas, and found that the melanoma cells were less sensitive to $\mathrm{x}$-ray irradiation if they had a p16 mutation. ${ }^{69}$
Therefore, an adjuvant therapy targeting the expression of mutant p16 would not only lead to better cell cycle regulation, but render the melanoma cells more radiosensitive.

A recent randomized trial of ipilimumab evaluated the role of an antibody targeting CTLA-4 in HLA-A*0201-positive unresectable stage III/IV melanoma patients who progressed on chemotherapy, and showed a significant improvement in median overall survival (10.0 months versus 6.4 months, $P<0.0001) .{ }^{70}$ Radiotherapy effects on the cellular immune system, or abscopal effects, may be partially responsible for the apparent positive impact on node-positive melanoma. Irradiated tumor tissues may develop inflammation leading to improved immune system activation, and how this can be utilized to make the emerging biologics more effective is an area of ongoing research. ${ }^{71}$

\section{Areas for future research and growth \\ Concurrent chemoradiotherapy}

Currently, the only approved chemotherapy for metastatic melanoma is the oral equivalent of dacarbazine, ie, temozolomide, which has a response rate of about $10 \%$ and a median survival of 8-9 months. ${ }^{72}$ Concurrent use of temozolomide with radiotherapy is the standard care for patients with glioblastoma multiforme, because this combined approach has shown increased efficacy over either approach alone. The combined approach of temozolomide given concurrently with radiation therapy has been tested for melanoma with mixed results. ${ }^{73}$

\section{Concurrent immunoradiotherapy}

Other approved agents for advanced melanoma include highdose interleukin-2, but only one in 20 patients receives lasting benefits with the use of interleukin-2. ${ }^{72}$ Interleukins are cytokines, similar to interferons (IFNs), in that they are potent modulators of the immune system. IFN- $\alpha$, a type I IFN, frequently used as adjuvant therapy for melanoma, is synthesized in vivo to respond to a viral infection and to kill tumor cells. ${ }^{74}$ By binding to its cell receptor, IFN- $\alpha$ activates the transcription factor ISGF3, which then activates the IFN-stimulated response element in the promoter sequence of the $\mathrm{p} 53$ gene. Activation of the IFN-stimulated response element induces p53 production, leading to increased apoptosis of the tumor cells. ${ }^{75,76}$ Adjuvant IFN can improve relapse-free survival in high-risk patients and patients with low-risk to intermediaterisk disease without impacting overall survival. ${ }^{77}$ Improvements in local recurrence without impact on overall survival have also been demonstrated in other studies. ${ }^{51,78}$ Only one 
study of 287 patients with melanomas $>4.0 \mathrm{~mm}$ and/or one regional metastasis randomized to either adjuvant therapy with high-dose IFN- $\alpha 2 b$ for 1 year or to observation, demonstrated an improvement in both disease-free survival (1.0 years versus 1.7 years $P=0.0023)$ and overall survival $(2.8$ years versus 3.8 years, one-sided $P=0.0237$ ) at 6.9 years of follow-up. ${ }^{79}$ This was not replicated in a subsequent randomized trial by the same group for stage IIB and III patients. ${ }^{80}$ Consequently, the use of IFN as an adjuvant therapy is under debate for patients who have progressed to Stage II or beyond.

Case reports have shown significant tumor regression when IFN therapy was combined concurrently with radiotherapy for large unresectable sinonasal melanoma. ${ }^{81}$ The combination approach has raised concerns about increased toxicity. ${ }^{82,83}$ One small study of 10 patients demonstrated a possible increase in subacute and late complications when radiation is delivered concurrently with IFN therapy ${ }^{84}$ However, this concern is not shared by others who strongly advocate evaluating a combined approach in future protocols, ${ }^{85}$ and state that concurrent radiation and IFN therapy may be safely delivered under appropriate clinical monitoring. ${ }^{82}$

\section{Concurrent bioradiotherapy}

Several clinical advances in the past decade have led to new treatment strategies, including agents targeted against mutations in B-Raf (PLX4032 and sorafenib) and c-KIT (kinase inhibitors such as imatinib), T cell immunotherapy, removal of immunologic inhibition at checkpoints in $\mathrm{T}$ cell activation (anti-CTLA 4 antibody), and many others. ${ }^{72,86}$ Many of these compounds have yet to be combined with radiation in the clinical setting, and represent an area of future research.

Recent studies in mouse models have suggested that radiation is more effective when combined with immunotherapy. ${ }^{87}$ A study by Lugade et al demonstrated that radiation to tumors in mice resulted in an increase in IFN- $\gamma$, allowing for a more effective cytotoxic $\mathrm{T}$ cell-mediated response against melanoma tumor cells. The authors concluded that radiation improved the lytic sensitivity of the tumor cells to cytotoxic T cells by increasing apoptosis-inducing STAT1 activation, and suggested that localized irradiation should be combined with emerging immunotherapy in the future. ${ }^{87}$ Similarly, another recent preclinical study demonstrated that radiation is a powerful agent in improving synthetic dsRNA therapy for an in vivo mouse melanoma model. ${ }^{88}$ Lastly, a recent study by Khan et al demonstrated that riluzole, an inhibitor of glutamate release by human GRM1-expressing melanoma cells, enhanced radiosensitivity in melanoma cells in vitro and in vivo. ${ }^{89}$ Khan et al currently have a Phase I protocol underway for patients with melanoma brain metastases to test the role of combining riluzole with whole brain radiation.

\section{Conclusion}

Although it appears true that malignant melanoma is a relatively radioinsensitive tumor type, the use of favorable fractionation schemes and treatment delivery plans combined with greater radiobiological understanding may provide increased local control in certain clinical situations. Intracranial radiosurgery and stereotactic radiotherapy appear to provide control rates for melanoma on a par with that seen for other more sensitive epithelial tumors. Small and medium choroidal melanomas show a natural history in which metastasis outside the eye is unusual, thus validating the apparent safety of protracted tumor response to brachytherapy which produces acceptable levels of visual field sparing as an alternative to surgical enucleation. Finally, recent Phase III data have shown significant improvements in local control after lymphatic radiotherapy for recurrent node-positive post-lymphadenectomy patients. For all of these clinical scenarios, it appears that a concentrated highly targeted radiation dose, with minimal fractionation and normal tissue inclusion, represents some of the keys to restoring confidence in the importance of this therapeutic modality for malignant melanoma.

Recent molecular biological understanding suggests that malignant melanoma deposits, long known to proliferate primarily in ultraviolet-exposed body surfaces, have evolved multiple overlapping protective mechanisms capable of activating or suppressing master gene panels controlling the dynamics of cell cycle progression in the face of cellular or chromosomal damage. Among these top-line damage sensor and repair pathways are p53, ATM, $\mathrm{NF}-\kappa \mathrm{B}$, and components of the cyclin/cyclin-dependent kinase regulatory network. The reversal or slowing of some of these protective pathways via genetic modifications (eg, targeted mutations) or pharmacologic inhibitors (eg, p53 or p16 small molecule inhibitors) perhaps in combination with appropriately fractionated radiotherapy and perhaps immunotherapy and chemotherapy may finally transform melanoma from the most lethal to one of the most curable of the primary cutaneous malignancies.

\section{Acknowledgments}

We would like to thank Mr Gaurav Choudhary (Case Western Reserve University) for help with illustrations and the National Institutes of Health for partial support to AA. 


\section{Disclosure}

The authors report no conflicts of interest in this work.

\section{References}

1. Algazi AP, Soon CW, Daud AI. Treatment of cutaneous melanoma: Current approaches and future prospects. Cancer Manag Res. 2010; 2:197-211.

2. Mouawad R, Sebert M, Michels J, Bloch J, Spano JP, Khayat D. Treatment for metastatic malignant melanoma: old drugs and new strategies. Crit Rev Oncol Hematol. 2010;74:27-39.

3. Jilaveanu LB, Aziz SA, Kluger HM. Chemotherapy and biologic therapies for melanoma: Do they work? Clin Dermatol. 2009;27:614-625.

4. MacKee GM, Cipollaro AC, Montgomery H. X-rays and Radium in the Treatment of Diseases of the Skin. 4th ed. Philadelphia, PA: Lea and Febiger; 1946.

5. Million RR, Cassisi NJ. Management of Head and Neck Cancer: A Multidisciplinary Approach. Philadelphia, PA: Lippincott; 1984.

6. Jenrette JM. Malignant melanoma: the role of radiation therapy revisited. Semin Oncol. 1996;23:759-762.

7. Stevens G, McKay MJ. Dispelling the myths surrounding radiotherapy for treatment of cutaneous melanoma. Lancet Oncol. 2006; 7:575-583.

8. Overgaard J, Overgaard M, Hansen PV, von der Maase H. Some factors of importance in the radiation treatment of malignant melanoma. Radiother Oncol. 1986;5:183-192.

9. Rofstad EK. Radiation biology of malignant melanoma. Acta Radiol Oncol. 1986;25:1-10

10. Korcum AF, Sanlioglu S, Aksu G, Tuncel N, Erin N. Radiotherapyinduced decreases in substance $\mathrm{P}$ levels may potentiate melanoma growth. Mol Med Report. 2009;2:319-326.

11. Homer MJ, Ries LAG, Krapcho M, et al. SEER Cancer Statistics Review,1975-2006. Bethseda, MD: National Cancer Institute; 2009. Available from: http://seer.cancer.gov/csr/1975_2006/. Accessed April 3,2010 .

12. Jemal A, Siegel R, Ward E, Hao Y, Xu J, Thun MJ. Cancer statistics, 2009. CA Cancer J Clin J. 2009;59:225-249.

13. Balch CM, Gershenwald JE, Soong SJ, et al. Final version of 2009 AJCC melanoma staging and classification. J Clin Oncol. 2009; 27:6199-6206.

14. Rigel DS, Carucci JA. Malignant melanoma: Prevention, early detection, and treatment in the 21 st century. CA Cancer J Clin. 2000; 50:215-236.

15. Network NCC. NCCN Clinical Practice Guidelines in Oncology: Melanoma. 2010. Available from: http://www.nccn.org/professionals/ physician_gls/f_guidelines.asp. Accessed June 22, 2011.

16. Khayat D, Rixe O, Martin G, et al. Surgical margins in cutaneous melanoma ( $2 \mathrm{~cm}$ versus $5 \mathrm{~cm}$ for lesions measuring less than 2.1-mm thick). Cancer. 2003;97:1941-1946.

17. Balch CM, Soong SJ, Smith T, et al. Long-term results of a prospective surgical trial comparing $2 \mathrm{~cm}$ vs $4 \mathrm{~cm}$ excision margins for 740 patients with 1-4 mm melanomas. Ann Surg Oncol. 2001;8:101-108.

18. Veronesi U, Cascinelli N. Narrow excision (1-cm margin). A safe procedure for thin cutaneous melanoma. Arch Surg. 1991;126:438-441.

19. Veronesi U, Cascinelli N, Adamus J, et al. Thin stage I primary cutaneous malignant melanoma. Comparison of excision with margins of 1 or $3 \mathrm{~cm}$. N Engl J Med. 1988;318:1159-1162.

20. Thomas JM, Newton-Bishop J, A'Hern R, et al. Excision margins in high-risk malignant melanoma. $N$ Engl J Med. 2004;350:757-766.

21. Coley WB, Leucutia T. The treatment of metastatic tumors of the skin: pigmented moles and melanomas. AJR Am J Roentgenol. 1924;11:335-336.

22. Ellis F. Radiosensitivity of malignant melanomata. Br J Radiol. 1939; $12: 327-352$

23. Hellriegel W. Radiation therapy for primary and metastatic melanoma. Ann NY Acad Sci. 1963;100:131-141.
24. Barranco SC, Romsdahl MM, Humphrey RM. The radiation response of human malignant melanoma cells grown in vitro. Cancer Res. 1971;31:830-833.

25. Overgaard J, von der Maase H, Overgaard M. A randomized study comparing two high-dose per fraction radiation schedules in recurrent or metastatic malignant melanoma. Int J Radiat Oncol Biol Phys. 1985;11:1837-1839.

26. Sause WT, Cooper JS, Rush S, et al. Fraction size in external beam radiation therapy in the treatment of melanoma. Int J Radiat Oncol Biol Phys. 1991;20:429-432.

27. Bentzen SM, Overgaard J, Thames HD, et al. Clinical radiobiology of malignant melanoma. Radiother Oncol. 1989;16:169-182.

28. Konefal JB, Emami B, Pilepich MV. Malignant melanoma: analysis of dose fractionation in radiation therapy. Radiology. 1987;164:607-610.

29. Ang KK, Byers RM, Peters LJ, et al. Regional radiotherapy as adjuvant treatment for head and neck malignant melanoma. Preliminary results. Arch Otolaryngol Head Neck Surg. 1990;116:169-172.

30. Burmeister BH, Mark Smithers B, Burmeister E, et al. A prospective phase II study of adjuvant postoperative radiation therapy following nodal surgery in malignant melanoma-Trans Tasman Radiation Oncology Group (TROG) Study 96.06. Radiother Oncol. 2006;81:136-142.

31. Henderson MA, Burmeister B, Thompson JF, et al. Adjuvant radiotherapy and regional lymph node field control in melanoma patients after lymphadenectomy: results of an Intergroup randomized trial (ANZMTG 01.02/TROG 02.01). J Clin Oncol. 2009;27(18 Suppl):Abstr LBA9084

32. Skibber JM, Soong SJ, Austin L, Balch CM, Sawaya RE. Cranial irradiation after surgical excision of brain metastases in melanoma patients. Ann Surg Oncol. 1996;3:118-123.

33. Sampson JH, Carter JHJ, Friedman AH, Seigler HF. Demographics, prognosis, and therapy in 702 patients with brain metastases from malignant melanoma. J Neurosurg. 1998;88:11-20.

34. Mori Y, Kondziolka D, Flickinger JC, Kirkwood JM, Agarwala S, Lunsford LD. Stereotactic radiosurgery for cerebral metastatic melanoma: factors affecting local disease control and survival. Int J Radiat Oncol Biol Phys. 1998;42:581-589.

35. Selek U, Chang EL, Hassenbusch S Jr, et al. Stereotactic radiosurgical treatment in 103 patients for 153 cerebral melanoma metastases. Int J Radiat Oncol Biol Phys. 2004;59:1097-1106.

36. Yu C, Chen JC, Apuzzo ML, et al. Metastatic melanoma to the brain: prognostic factors after gamma knife radiosurgery. Int J Radiat Oncol Biol Phys. 2002;52:1277-1287.

37. Radbill AE, Fiveash JF, Falkenberg ET, et al. Initial treatment of melanoma brain metastases using gamma knife radiosurgery: an evaluation of efficacy and toxicity. Cancer. 2004;101:825-833.

38. Scorsetti M, Facoetti A, Navarria P, et al. Hypofractionated stereotactic radiotherapy and radiosurgery for the treatment of patients with radioresistant brain metastases. Anticancer Res. 2009;29:4259-4263.

39. Stone A, Cooper J, Koenig KL, Golfinos JG, Oratz R. A comparison of survival rates for treatment of melanoma metastatic to the brain. Cancer Invest. 2004;22:492-497.

40. Herlyn M, Nathanson KL. Taking the guesswork out of uveal melanoma. N Engl J Med. 2010;363:2256-2257.

41. Van Raamsdonk JM, Meng Y, Camp D, et al. Decreased energy metabolism extends life span in Caenorhabditis elegans without reducing oxidative damage. Genetics. 2010;185:559-571.

42. Van Raamsdonk CD, Griewank KG, Crosby MB, et al. Mutations in GNA11 in uveal melanoma. N Engl J Med. 2010;363:2191-2199.

43. Diener-West M, Earle JD, Fine SL, et al. The COMS randomized trial of iodine 125 brachytherapy for choroidal melanoma, III: Initial mortality findings. COMS Report No. 18. Arch Ophthalmol. 2001;119:969-982.

44. Temam S, Mamelle G, Marandas P, et al. Postoperative radiotherapy for primary mucosal melanoma of the head and neck. Cancer. 2005; $103: 313-319$ 
45. Owens JM, Roberts DB, Myers JN. The role of postoperative adjuvant radiation therapy in the treatment of mucosal melanomas of the head and neck region. Arch Otolaryngol Head Neck Surg. 2003;129: 864-868.

46. Curtin JA, Busam K, Pinkel D, Bastian BC. Somatic activation of KIT in distinct subtypes of melanoma. J Clin Oncol. 2006;24: 4340-4346.

47. Handolias D, Hamilton AL, Salemi R, et al. Clinical responses observed with imatinib or sorafenib in melanoma patients expressing mutations in KIT. Br J Cancer. 2010;102:1219-1223.

48. Moncrieff MD, Martin R, O’Brien CJ, et al. Adjuvant postoperative radiotherapy to the cervical lymph nodes in cutaneous melanoma: Is there any benefit for high-risk patients? Ann Surg Oncol. 2008; 15:3022-3027.

49. Ballo MT, Ang KK. Radiotherapy for cutaneous malignant melanoma: Rationale and indications. Oncology (Williston Park). 2004;18: 99-107.

50. Chang DT, Amdur RJ, Morris CG, Mendenhall WM. Adjuvant radiotherapy for cutaneous melanoma: Comparing hypofractionation to conventional fractionation. Int J Radiat Oncol Biol Phys. 2006; 66:1051-1055.

51. Verma S, Quirt I, McCready D, Bak K, Charette M, Iscoe N. Systematic review of systemic adjuvant therapy for patients at high risk for recurrent melanoma. Cancer. 2006;106:1431-1442.

52. Agrawal S, Kane JM 3rd, Guadagnolo BA, Kraybill WG, Ballo MT. The benefits of adjuvant radiation therapy after therapeutic lymphadenectomy for clinically advanced, high-risk, lymph node-metastatic melanoma. Cancer. 2009;115:5836-5844.

53. Ballo MT, Ross MI, Cormier JN, et al. Combined-modality therapy for patients with regional nodal metastases from melanoma. Int J Radiat Oncol Biol Phys. 2006;64:106-113.

54. Uong A, Zon LI. Melanocytes in development and cancer. $J$ Cell Physiol. 2010;222:38-41.

55. Miller AJ, Mihm MC Jr. Melanoma. N Engl J Med. 2006;355: 51-65.

56. Satyamoorthy K, Chehab NH, Waterman MJ, et al. Aberrant regulation and function of wild-type p53 in radioresistant melanoma cells. Cell Growth Differ. 2000;11:467-474.

57. Crosby ME, Almasan A. Opposing roles of E2Fs in cell proliferation and death. Cancer Biol Ther. 2004;3:1208-1211.

58. Almasan A, Yin Y, Kelly RE, et al. Deficiency of retinoblastoma protein leads to inappropriate S-phase entry, activation of E2Fresponsive genes, and apoptosis. Proc Natl Acad Sci U SA. 1995;92: 5436-5440.

59. Mazumder S, DuPree EL, Almasan A. A dual role of cyclin E in cell proliferation and apoptosis may provide a target for cancer therapy. Curr Cancer Drug Targets. 2004;4:65-75.

60. Fecher LA, Cummings SD, Keefe MJ, Alani RM. Toward a molecular classification of melanoma. J Clin Oncol. 2007;25:1606-1620.

61. Nogueira C, Kim KH, Sung H, et al. Cooperative interactions of PTEN deficiency and RAS activation in melanoma metastasis. Oncogene. 2010;29:6222-6232.

62. Haferkamp S, Becker TM, Scurr LL, Kefford RF, Rizos H. p16INK 4a-induced senescence is disabled by melanoma-associated mutations. Aging Cell. 2008;7:733-745.

63. Johnson SM, Torrice CD, Bell JF, et al. Mitigation of hematologic radiation toxicity in mice through pharmacological quiescence induced by CDK4/6 inhibition. J Clin Invest. 2010;120:2528-2536.

64. Tsai J, Lee JT, Wang W, et al. Discovery of a selective inhibitor of oncogenic B-Raf kinase with potent antimelanoma activity. Proc Nat Acad Sci U S A. 2008;105:3041-3046.

65. Flaherty KT, Puzanov I, Kim KB, et al. Inhibition of mutated, activated BRAF in metastatic melanoma. $N$ Engl $J$ Med. 2010;363: 809-819.

66. Gudkov AV, Komarova EA. Radioprotection: smart games with death. J Clin Invest. 2010;120:2270-2273.
67. Yang J, Splittgerber R, Yull FE, et al. Conditional ablation of Ikkb inhibits melanoma tumor development in mice. J Clin Invest. 2010; 120:2563-2574.

68. Johnson GE, Ivanov VN, Hei TK. Radiosensitization of melanoma cells through combined inhibition of protein regulators of cell survival. Apoptosis. 2008;13:790-802.

69. Matsumura Y, Yamagishi N, Miyakoshi J, Imamura S, Takebe H. Increase in radiation sensitivity of human malignant melanoma cells by expression of wild-type p16 gene. Cancer Lett. 1997;115:91-96.

70. Hodi FS, O’Day SJ, McDermott DF, et al. Improved survival with ipilimumab in patients with metastatic melanoma. $N$ Engl $\mathrm{J} \mathrm{Med}$. 2010;363:711-723.

71. Kaminski JM, Shinohara E, Summers JB, Niermann KJ, Morimoto A, Brousal J. The controversial abscopal effect. Cancer Treat Rev. 2005;31:159-172.

72. Ascierto PA, Streicher HZ, Sznol M. Melanoma: A model for testing new agents in combination therapies. J Transl Med. 2010;8:38.

73. Schild SE, Behl D, Markovic SN, et al. Brain metastases from melanoma: Is there a role for concurrent temozolomide in addition to whole brain radiation therapy? Am J Clin Oncol. 2010;33: 633-636.

74. Gray RJ, Pockaj BA, Kirkwood JM. An update on adjuvant interferon for melanoma. Cancer Control. 2002;9:16-21.

75. Liu YJ. IPC: professional type 1 interferon-producing cells and plasmacytoid dendritic cell precursors. Annu Rev Immunol. 2005; 23:275-306

76. Takaoka A, Hayakawa S, Yanai H, et al. Integration of interferon-alpha/ beta signalling to p53 responses in tumour suppression and antiviral defence. Nature. 2003;424:516-523.

77. Cascinelli N, Belli F, MacKie RM, Santinami M, Bufalino R, Morabito A. Effect of long-term adjuvant therapy with interferon alpha-2a in patients with regional node metastases from cutaneous melanoma: a randomised trial. Lancet. 2001;358:866-869.

78. Hancock BW, Wheatley K, Harris S, et al. Adjuvant interferon in high-risk melanoma: The AIM HIGH Study - United Kingdom Coordinating Committee on Cancer Research randomized study of adjuvant low-dose extended-duration interferon alfa-2a in high-risk resected malignant melanoma. J Clin Oncol. 2004;22: 53-61.

79. Kirkwood JM, Strawderman MH, Ernstoff MS, Smith TJ, Borden EC, Blum RH. Interferon alfa-2b adjuvant therapy of high-risk resected cutaneous melanoma: The Eastern Cooperative Oncology Group Trial EST 1684. J Clin Oncol. 1996;14:7-17.

80. Kirkwood JM, Ibrahim JG, Sondak VK, et al. High- and low-dose interferon alfa-2b in high-risk melanoma: First analysis of Intergroup trial E1690/S9111/C9190. J Clin Oncol. 2000;18:2444-2458.

81. Nguyen NP, Sallah S, Childress C, Salehpour MR, Karlsson U. Interferon-alpha combined with radiotherapy in the treatment of unresectable melanoma. Cancer Invest. 2001;19:261-265.

82. Gyorki DE, Ainslie J, Joon ML, Henderson MA, Millward M, McArthur GA. Concurrent adjuvant radiotherapy and interferon$\mathrm{alpha} 2 \mathrm{~b}$ for resected high risk stage III melanoma - a retrospective single centre study. Melanoma Res. 2004;14:223-230.

83. Conill C, Jorcano S, Domingo-Domenech J, et al. Toxicity of combined treatment of adjuvant irradiation and interferon alpha2b in high-risk melanoma patients. Melanoma Res. 2007;17:304-309.

84. Hazard LJ, Sause WT, Noyes RD. Combined adjuvant radiation and interferon-alpha 2B therapy in high-risk melanoma patients: the potential for increased radiation toxicity. Int J Radiat Oncol Biol Phys. 2002;52:796-800

85. Paul E, Muller I, Renner H, Bodeker RH, Cochran AJ. Treatment of locoregional metastases of malignant melanomas with radiotherapy and intralesional beta-interferon injection. Melanoma Res. 2003;13:611-617.

86. Mansfield AS, Markovic SN. Novel therapeutics for the treatment of metastatic melanoma. Future Oncol. 2009;5:543-557. 
87. Lugade AA, Sorensen EW, Gerber SA, Moran JP, Frelinger JG, Lord EM. Radiation-induced IFN-gamma production within the tumor microenvironment influences antitumor immunity. J Immunol. 2008; 180:3132-3139.

88. Le UM, Kaurin DG, Sloat BR, Yanasarn N, Cui Z. Localized irradiation of tumors prior to synthetic dsRNA therapy enhanced the resultant anti-tumor activity. Radiother Oncol. 2009;90:273-279.
89. Khan AJ, Wall BA, Ahlawat S, et al. Riluzole enhances ionizing radiation-induced cytotoxicity in human melanoma cells that ectopically express metabotropic glutamate receptor 1 in vitro and in vivo. Clin Cancer Res. 2011;17:1807-1814.

\section{Publish your work in this journal}

OncoTargets and Therapy is an international, peer-reviewed, open access journal focusing on the pathological basis of all cancers, potential targets for therapy and treatment protocols employed to improve the management of cancer patients. The journal also focuses on the impact of management programs and new therapeutic agents and protocols on patient perspectives such as quality of life, adherence and satisfaction. The manuscript management system is completely online and includes a very quick and fair peer-review system, which is all easy to use. Visit http://www.dovepress.com/testimonials.php to read real quotes from published authors.

Submit your manuscript here: http://www.dovepress.com/oncotargets-and-therapy-journal 